\title{
Optimizing Cost of Continuous Overlapping Queries over Data Streams by Filter Adaption
}

\author{
Qing Xie, Xiangliang Zhang, Member, IEEE, Zhixu Li, and Xiaofang Zhou, Senior Member, IEEE
}

\begin{abstract}
The problem we aim to address is the optimization of cost management for executing multiple continuous queries on data streams, where each query is defined by several filters, each of which monitors certain status of the data stream. Specially the filter can be shared by different queries and expensive to evaluate. The conventional objective for such a problem is to minimize the overall execution cost to solve all queries, by planning the order of filter evaluation in shared strategy. However, in streaming scenario, the characteristics of data items may change in process, which can bring some uncertainty to the outcome of individual filter evaluation, and affect the plan of query execution as well as the overall execution cost. In our work, considering the influence of the uncertain variation of data characteristics, we propose a framework to deal with the dynamic adjustment of filter ordering for query execution on data stream, and focus on the issues of cost management. By incrementally monitoring and analyzing the results of filter evaluation, our proposed approach can be effectively adaptive to the varied stream behavior and adjust the optimal ordering of filter evaluation, so as to optimize the execution cost. In order to achieve satisfactory performance and efficiency, we also discuss the trade-off between the adaptivity of our framework and the overhead incurred by filter adaption. The experimental results on synthetic and two real data sets (traffic and multimedia) show that our framework can effectively reduce and balance the overall query execution cost and keep high adaptivity in streaming scenario.
\end{abstract}

Index Terms-Overlapping queries, cost optimization, filter adaption, data streams

\section{INTRODUCTION}

$\mathrm{T}$ HE optimization of continuous query has been applied and studied widely in data stream management system [1], which benefits various applications such as real time medical care [2] and financial data monitoring [3]. We address multiple queries that are answered by combining the evaluation results of several filters, each of which monitors a specific status of data stream. It is worth noting that filters are often shared among different queries. Such kind of overlapping query is able to express sophisticated conditions, and thus has been commonly involved in practical applications. For example:

- In smart city road network, there can be hundreds of sensors deployed on different roads, which monitor the traffic status of the city all the time. Complex queries are requested based on the combination of different sensors to explore the interesting events of the traffic.

- Multimedia streams in Internet have attracted much attention nowadays, and one of the most significant tasks is the online detection of high level semantic events, which can be defined by the combination of basic characteristics of multimedia data.

- Q.Xie and X.Zhang ${ }^{\square}$ are with the Division of CEMSE, King Abdullah University of Science and Technology, Thuwal, Saudi Arabia. E-mail: \{Qing.Xie, Xiangliang.Zhang\}@kaust.edu.sa

- Z.Li is with the School of Computer Science and Technology, Soochow University, China.

Email: zhixuli@suda.edu.cn

- X.Zhou is with the School of ITEE, the University of Queensland, Brisbane, Australia, and the School of Computer Science and Technology, Soochow University, China

E-mail: zxf@itee.uq.edu.au

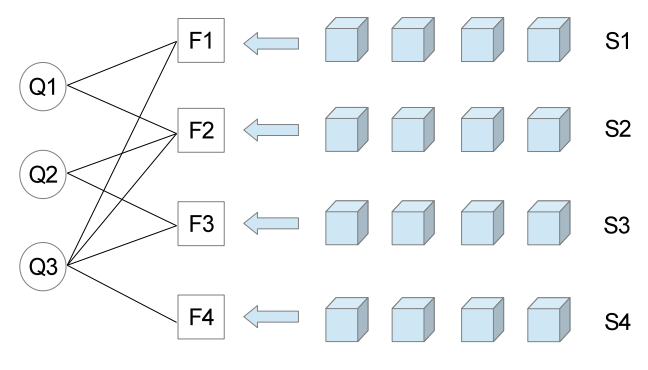

Fig. 1. Multiple queries with shared filters.

Figure 1 denotes a typical model of multiple queries with shared filters. Generally, a query is satisfied if all its involved filters are evaluated as "True", otherwise the query is unsatisfied, and the query execution cost is the sum cost of filters evaluated to determine the query result. Consider monitoring video streams and continuous queries are generated for event detection, e.g., find the scenes containing the meadow. Such kind of queries can be resolved as a conjunction of several filters, which detect the basic features of frame data, e.g., whether the pixel proportion of specific color is higher than a threshold. Usually hundreds of filters can be defined and shared among different queries.

Since the filters are usually expensive in evaluation, it is essential to improve the efficiency of query processing. Evidently, the overall cost of query execution can be effectively reduced by sharing the filter evaluation among different queries. We employ a simple instance to exemplify the shared query execution plan. For the case showed in Fig. 1, we assume that all filters will be evaluated as "False" for the current data items. If dealing with the filters in numerical order, we will first evaluate $F_{1}$, which can solve $Q_{1}$ and $Q_{3}$, and then $F_{2}$ to solve all queries. However, if we evaluate 
$F_{2}$ first, all queries can be answered immediately. Therefore the filter order is important in shared query execution.

Effective approaches have been proposed in previous works [4], [5] to design the filter evaluation ordering. More specifically, three important factors about a filter have been identified to determine the filter ordering:

- Cost: the cost to evaluate a filter $F_{i}, \operatorname{cost}\left(F_{i}\right)=c_{i}$, e.g., the time cost. Intuitively, to minimize the overall cost, filters with lower cost should be evaluated earlier.

- Selectivity: the probability that the data item satisfies a filter $F_{i}$, selectivity $\left(F_{i}\right)=s_{i}$. For effectively reducing the number of queries to be executed, filters with lower selectivity should be evaluated earlier to answer relevant queries.

- Popularity: the number of queries containing the filter $F_{i}$, popularity $\left(F_{i}\right)=p_{i}$. For answering as many queries as possible, it is intuitive to evaluate filters with larger popularity earlier.

In our work, besides these three factors, we further consider an important characteristics of data streams, the variation. In real streaming scenario, the variation in data characteristics, e.g., the change of video scene, can potentially cause changes in the selectivity factor and further influence the filter ordering. For example, if the selectivity of $F_{2}$ becomes close to 1, i.e., it has fair chance of obtaining "True" result, the priority of $F_{2}$ in the evaluation ordering should be dramatically dropped. Such variation makes the filter ordering complicated in real processing, and therefore the evaluation plan should be carried out in an adaptive way for coping with the variation of streaming data.

The existing works in [4], [5] model the query execution as probabilistic set covering [6], and focus on the three factors mentioned above to design the filter ordering. With the assumption that the data characteristics are always stable, the approaches of [4], [5] optimize the execution cost only with fixed and predetermined values of $c_{i}, s_{i}$ and $p_{i}$. However, in the practical streaming scenario, the potential change in the filter selectivity caused by data variation will make these approaches invalid. Therefore, we target on planning the order of filter evaluation in shared strategy with changing selectivity. To achieve this, we propose a new filter adaption mechanism, which includes filter monitoring and updating modules and carefully controls the cost distribution in filter adaption.

The monitoring module records the current data status and detects the potential change in the data characteristics. It is difficult to define a general and universal criterion to check the stream variation by directly analyzing the data items, since the streams can have different data types and characteristics. Thus, we propose to focus on the filter evaluation results and aim to discover certain indicators that are more general and independent to specific data characteristics, to examine the potential change in selectivity factor. If the selectivity change is detected, the updating module will be activated to recalculate the selectivity value by a statistical approach, which best estimates the new selectivity of a filter based on its recent evaluation results.
These two modules are essential to the treatment of selectivity variation. However, extra cost may be required to maintain the filter evaluation results in addition to regular query execution plan. The cost management in the filter adaption thus becomes sensitive. Our goal is to optimize the overall cost of query execution by paying a limited extra price on filter adaption. In order to restrict the additional cost for filter monitoring and selectivity estimation, we assign different priorities to the filters according to their influence in overall query execution. In this way, the extra cost will be distributed based on the priorities. It is even more noteworthy that the additional overhead in filter adaption can benefit the reduction of overall query execution cost, so there exists a trade-off between extra overhead and the cost saving in overall query execution. We take efforts to analyze the balance of these two aspects and explore the effective way of cost management.

We summarize the contributions of our work as follows:

- We study the optimization of overlapping queries execution with shared filters in streaming scenario, where the data characteristics are potentially changing to affect the filter selectivity.

- We design a framework to monitor the data status and update filter factors, so as to make the query execution adaptive to the actual stream variation.

- We discuss the cost management in the filter adaption and show that the performance and efficiency can be balanced by tuning the overhead in filter adaption.

- The experimental evaluation indicates that our solution has general meaning for query optimization in stream processing, and can be applied in real application scenarios such as traffic and multimedia data.

The following part of our paper is organized as below: In Section 2, we introduce the most relevant works in literature. Following some preliminary concepts and definitions provided in Section 3, we describe our strategy and framework in details in Section 4 and Section 5 respectively. The experimental evaluation is provided in Section 6. Finally we conclude our work in Section 7.

\section{Related Works}

Publish-subscribe system [7], [8], [9], [10] has been studied since the 1990', which can be modeled as continuous queries with shared filters over data stream. In this kind of system, different users define different requirements, or subscriptions, on incoming data stream, which is evaluated continuously to match these subscriptions. The publishsubscribe system mainly focuses on three points: to analyze the relationship of subscriptions and find the sharing structure; to support millions of queries, since it would be applied on large scale Internet application; to use diskbased indexing to decrease memory and IO cost. Therefore, the pub-sub system does not consider the order of filter evaluation, which is the main concern in our problem.

The analysis of common subexpressions [11] is discussed and used for multiple query optimization. Dynamic programming algorithm has been proposed to select the access 
plan for each query considering the sharing of subexpressions, and achieve the minimum processing cost [12]. The concept of subexpression is similar to filter, but it is a fix task of the access plan for a query other than a probabilistic condition. The challenge of this problem is how to globally select access plan for each query among all alternatives.

The research of shared filter execution is also related with the continuous query [13], [14], [15] in DSMS [1]. Conventional continuous query problem in DSMS engages a query sequence that matches the incoming data stream or time series database [16], [17]. Based on that, more complicated query types are defined and discussed, and researchers focus more on optimization of evaluation cost [18]. Continuous join is discussed in [19], [20] which aim to find the common subexpressions for join operation in streaming data. Window aggregation is a common problem in streaming scenario and engaged in [21], [22], which deal with sharing issue but only for cheap filters.

Pipelined filter ordering [23], [24], [25], [26] addresses the ordering of expensive filters for query optimization, which is similar to our problem setting. However, this problem only considers single query, so our problem model is a natural extension that takes in to account the relationship of all filters and queries to optimize filter ordering.

As mentioned in Section 1, the shared filter ordering problem can be modeled as a probabilistic set cover [6], that is, a set can cover different number of elements with certain probability, which corresponds to the probability that filter can be true or false. As a classical NP-hard problem, ILP (Integer Linear Programming) approach can be applied to derive an approximate solution [27], which however, cannot be applied for continuous processing. For the sake of efficiency, greedy algorithms are more favorable for NP-hard problem. Goemans et al. [28] proposed effective algorithms to solve stochastic covering problem, and achieved an napproximation ( $\mathrm{n}$ is the number of elements).

The optimization of queries with shared filters in data stream system is formally defined in [4], [5]. Effective greedy algorithms have been proposed to find the approximate solutions. Munagala et al. [4] designed a decision tree to determine the order of filter evaluation. The priority of a filter is determined by the expected cost on it to solve unit number of query. Liu et al. [5] model the relationship of queries and filters as a graph, and evaluating the edges that can potentially be covered by a filter. The priority of a filter is proportional to the expected cost on unit number of edges it can potentially cover.

The problem we address is different from that in [4], [5], and focuses more on the variation of streaming scenario. Our core attention is to analyze the filter results and the variation of data characteristics, and design efficient and adaptive filter evaluation. With the favorable outcomes of set cover and filter ordering problems, our work will discuss more on the adaptivity and the overhead issues.

\section{Preliminaries}

Formally, the query set can be denoted as $\mathcal{Q}=$ $\left\{Q_{1}, Q_{2}, \ldots, Q_{m}\right\}$, and the corresponding filter set is
$\mathcal{F}=\left\{F_{1}, F_{2}, \ldots, F_{n}\right\}$, each of which has three attributes: $\left\{c_{i}, s_{i}, p_{i}\right\}$ denoting evaluation cost, selectivity and popularity respectively. Each query is defined as the conjunction of several filters chosen from the filter set. If all the filters for a query are evaluated as "True", the query answer is "True"; otherwise if any filter is unsatisfied, the query is evaluated as "False".

The filters can be defined on multiple streams or a single stream, i.e., each filter monitors a data stream, or all filters are defined on one complex data stream. If not specified otherwise, we discuss multi-stream case, as the single stream case can be considered as a special case of multi-stream, where the variation in a single stream affects the selectivity of all filters at the same time. Without the loss of generality, we assume that the data items of different streams are received synchronously at a fixed sampling rate, and the queries are continuously evaluated as the stream proceeds. Within the scope of our work, the data items are mutually independent ${ }^{1}$. For each group of data items at a certain time stamp, we need a specific strategy to execute all the queries in $\mathcal{Q}$ by ordering the filter evaluation, which considers the three factors $\left\{c_{i}, s_{i}, p_{i}\right\}$ of each filter, and aims to reduce the overall cost by sharing filter evaluation.

We apply the query execution strategy similar to the work based on Residual Graph [5]. Intuitively, we can model the relationship of filters and queries as a filter-query graph that is exemplified in Fig. 2. When a filter is evaluated, partial edges associated to it can be covered (the dashed edges in the Fig. 2), and we solve all queries if and only if all edges are covered. The basic idea of the execution strategy is, we determine the order of filter evaluation according to the expected cost on unit number of edges it can potentially cover. Specifically, for a filter $F_{i}, E^{T}$ stands for the number of covered edges if $F_{i}$ is "True" (Fig. 2(a)), and $E^{F}$ is the number of covered edges if $F_{i}$ is "False" (Fig. 2(b)), so the expected cost on unit number of edges is,

$$
\langle\bar{C}\rangle=\frac{c_{i}}{s_{i} * E^{T}+\left(1-s_{i}\right) * E^{F}} .
$$

The filter with lower $\langle\bar{C}\rangle$ value will be assigned a higher evaluation priority. During the execution process, the $\langle\bar{C}\rangle$ values of the temporarily unsolved filters will be continuously updated.

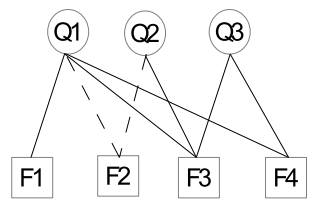

(a) F2=True, $E^{\top}=2$

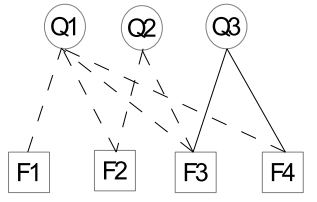

(b) F2=False, $E^{F}=6$

\section{Fig. 2. Graph model for filter-query relationship.}

In the streaming scenario, we aim to monitor the status of filter evaluation continuously, and dynamically update the filter selectivity if the stream variation is detected. The

1. Applications with dependency between data items will be further studied in future work. 
TABLE 1

List of Notations.

\begin{tabular}{|c|l|}
\hline Symbol & Description \\
\hline$Q, F$ & Query and Filter \\
\hline$c, s, p$ & Filter's cost, selectivity and popularity \\
\hline$E^{T}, E^{F}$ & The covered edges when filter is "True" and "False" \\
\hline$L$ & Window length \\
\hline$L_{o}$ & The observation number of filter evaluation \\
\hline $\mathbf{X}_{(t)}$ & The filter evaluation order vector at time $t$ \\
\hline$O_{(t)}$ & The filter evaluation outcome at time $t$ \\
\hline$\delta$ & The threshold for selectivity variation judgement \\
\hline$I$ & The filter's influence measure \\
\hline$p^{s}$ & The selection probability for filter extra sampling \\
\hline$p^{r}$ & The priority of filter extra sampling \\
\hline$p_{c}$ & The probability threshold controlling priority assignment \\
\hline
\end{tabular}

variation in data stream manifests in different forms, such as suddenly, incrementally, or gradually [29]. In all of these forms, streams behave stably for a relatively long period before and after one variation, e.g., stable with at least hundreds of samples that are statistically sufficient for representing a stable distribution. The filter evaluation results overing the streams are recorded by applying a sliding window to keep the updated information. Our approach will be based on all the updated information and answer the following essential questions:

- Q1: How to make the framework adaptive to the variation of data characteristics, i.e., automatically detect the change and update the filter selectivity, so as to adjust the evaluation plan.

- Q2: How to optimize the cost distribution and balance the execution overhead. The overhead includes the regular cost of filter evaluation and the extra cost in monitoring and updating modules.

The notations in this paper are summarized in Table 1.

\section{Framework of Filter Adaption}

In this section, we will describe the general framework named FilAda for filter adaption. We first introduce its monitoring and updating modules respectively, and then discuss the cost management in the updating module.

\subsection{Selectivity Monitoring}

Firstly, we interpret the applied sliding window in details. For each sampling time stamp, we can obtain the filter evaluation results after executing the sampled data group, and the results for each filter will be recorded into the sliding window. The sliding window will keep and update the results of recent $L$ samples in chronological order. By default, we set the window length as 200 . For each filter at each time stamp in the window, the basic information we can store includes and is not limited to ${ }^{2}$ :

- The filter evaluation cost;

- Whether the filter is evaluated or not;

2. Other useful information of filter evaluation can also be extended into the sliding window when necessary.
- The actual filter evaluation outcome.

Intuitively, the selectivity variation can be reflected by the fluctuation of overall query execution cost. If we detect bursting increase or drop to the execution cost records in the window, the filter selectivity distribution may have changed. Such a solution is straightforward but has several limitations. Firstly, the selectivity change may not cause salient variation in cost value. For example, after the change of selectivity distribution, all queries may be solved by different but similar amount of filter evaluations, and it will lead to almost same cost for entire query execution (assume that each filter has the same evaluation cost without the loss of generality). Secondly, the criterion of decision making is derived from the variation of cost value, so the result is sensitive to the threshold setting. For different filter-query relationships, the unique threshold value may cause false alarm or dismissal. Therefore, we propose to study the mechanism of filter evaluation strategy and explore more robust and reliable variables to reflect the selectivity status.

The query execution strategy dynamically selects filters to evaluate. We conjecture that for a given filter selectivity distribution, the order of filter evaluation is relatively stable. Since the filter-query graph has been determined in advance, if the selectivity keeps unchanged, the priority of the filter can be always identified by Equation (1). Although the actual filter ordering can be different for each data item, we can still expect the overall stable filter ordering, especially the relative priority of each filter. If the overall selectivity distribution has changed, the results of filter evaluation will be different from the expectation, which can potentially lead to the obviously varied filter evaluation order.

Based on the above analysis, during the overall query execution, we record the numerical evaluation order of each filter, so as to generate an evaluation order vector, which can also be recorded into the sliding window. For example, if we evaluate $F_{3}$ and then $F_{1}$ to solve all queries, the order vector for $\left\{F_{1}, F_{2}, F_{3}\right\}$ will be $\{2,0,1\}$. Fig. 3 exemplifies the actual filter order vectors in processing streaming data items, with 100 filters and 1200 samples. The vertical axis denotes different filters, and the gray level of each point stands for the evaluation order of a filter at certain time stamp. For these 1200 samples, the selectivity variation occurs after 1000. As demonstrated in the figure, we can discover salient difference in the filter evaluation order vectors, which is the indicator we propose to catch.

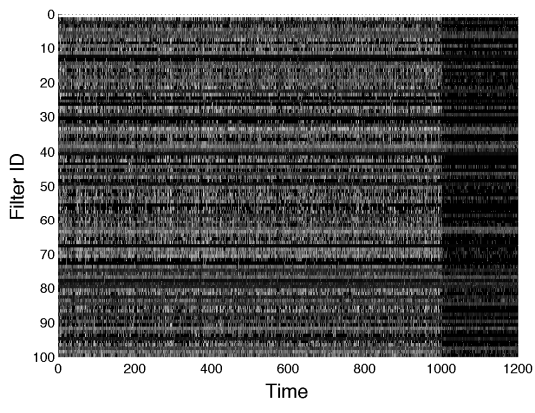

Fig. 3. The order vector of filter evaluation. 
To identify the salient variation of the high-dimensional order vectors, we propose to employ PCA (Principal Component Analysis) to analyze the overall distribution of the vectors in the sliding window. Intuitively, if the selectivity distribution is unchanged, the loading vectors ${ }^{3}$ of the principal components are relatively stable, otherwise there can be certain rotation to the loading vectors [30]. By this means, we are able to detect the stream variation by monitoring the principal components of evaluation order vectors.

More formally, we divide the sliding window into two parts with equal length, and denote the filter evaluation order vectors in the half windows as $\mathbf{X}^{1}$ and $\mathbf{X}^{2}$ respectively. Here, each $\mathbf{X}^{k}$ is a matrix with rows representing the evaluation order vectors, e.g., $\mathbf{X}_{(t)}^{1}=\left(x_{1}, x_{2}, \ldots, x_{n}\right)_{(t)}$ is the order vector at time stamp $t$ in the first half window, and $x_{i}$ is the evaluation order of $F_{i}$ (if $F_{i}$ is not evaluated, $x_{i}=0$ ). For each $\mathbf{X}^{k}$, we apply PCA and find the loading vector corresponding to the first principal component, which captures the maximal variance of the data. According to the theory of PCA, assume the first loading vector for $\mathbf{X}^{k}$ is $\mathbf{v}^{k}$, we have ${ }^{4}$ :

$$
\mathbf{v}^{k}=\underset{\|\mathbf{v}\|=1}{\arg \max } \sum_{t}\left(\mathbf{X}_{(t)}^{k} \cdot \mathbf{v}\right)^{2}, \quad k=1,2 .
$$

In fact, the first loading vector $\mathbf{v}^{k}$ also indicates the best linear fitting line that minimizes the squared perpendicular distance from each of the $\mathbf{X}_{(t)}^{k}$ to the line, $\sum_{t}\left\|\mathbf{X}_{(t)}^{k}-\left(\mathbf{X}_{(t)}^{k} \cdot \mathbf{v}^{k}\right) \mathbf{v}^{k}\right\|^{2}, k=1,2$. This fitting line, in parallel with $\mathbf{v}^{k}$, is known as Orthogonal Regression function. It is the best linear approximation of the data with minimum loss, and thus is often used to represent the data it approximates. The loading vector corresponding to the second PC is perpendicular to the first loading vector, and is in parallel with the residual vectors, $\mathbf{X}_{(t)}^{k}-\left(\mathbf{X}_{(t)}^{k} \cdot \mathbf{v}^{k}\right) \mathbf{v}^{k}$, which are vectors pointing from the projections (on to the first loading vector $\left.\mathbf{v}^{k}\right)$ to the original $\mathbf{X}_{(t)}^{k}$. This second loading vector is the best fitting to the residuals after subtracting off the first component. Recursively, the $j$ th principal component is the leading component of the residuals after subtracting off the first $j-1$ components. Therefore, we consider the usage of the loading vector corresponding to the first PC because it best represents $\mathbf{X}^{k}$ with minimum loss.

In order to quantify the difference of two loading vectors, we use cosine distance, which is widely used in processing high-dimensional data:

$$
D\left(\mathbf{v}^{1}, \mathbf{v}^{2}\right)=1-\frac{\left|\mathbf{v}^{1} \cdot \mathbf{v}^{2}\right|}{\left\|\mathbf{v}^{1}\right\|\left\|\mathbf{v}^{2}\right\|} .
$$

Since the result of cosine distance calculation is bounded within $[0,1]$, it can provide the normalized similarity measure and deliver more general sense. By defining a proper threshold $\delta$, we can claim the selectivity change if $D\left(\mathbf{v}^{1}, \mathbf{v}^{2}\right)>\delta$. In our work, we set $\delta=0.3$ by default.

3. Loading vectors are the basis vectors spanning the new orthogonal coordinate system from PCA analysis.

4. Here $\mathbf{X}_{(t)}^{k}$ is centered by subtracting the mean value.
PCA can provide an overall view on the evaluation order vectors, and act as a robust indicator to monitor the selectivity distribution. However, it is not always performing well in practice especially when the observation number is insufficient. Too small sample size may cause the instability of PCA, and at least one hundred observations are recommended in literature. In other words, a window of large enough size should be used to guarantee the performance. However, large window will bring inefficiency, since the cost of maintenance and storage will relatively increase.

In case that the filter number is beyond the capability of the sliding window for PCA processing, we are motivated to find an alternative solution. We propose to find a reference vector to represent the overall distribution of order vectors. The window is still divided into two equal parts, namely historic and recent parts, corresponding to $\mathbf{X}^{1}$ and $\mathbf{X}^{2}$. We expect that the order vectors in the historic part are relatively stable and the variation occurs in recent part. The reference vector is constructed by the mean vector of the historic part, so that each order vector can make equal contribution. Specifically, the reference vector is calculated as $\overline{\mathbf{x}}=\left(\bar{x}_{1}, \bar{x}_{2}, \ldots \bar{x}_{n}\right)$, where for $1 \leq i \leq n$, $\bar{x}_{i}=\sum_{t} x_{i(t)} / L^{\prime}$. Here, $x_{i(t)}$ is the evaluation order of $F_{i}$ at the time stamp $t$ in the historic window part, and $L^{\prime}=\frac{1}{2} L$ is the part length. Given the reference vector $\overline{\mathbf{x}}$, we calculate the pairwise cosine distance of it with the vectors in the recent part, and obtain the mean value of the distances accordingly. If the mean distance value is salient, we claim the occurrence of variation in overall selectivity distribution. The threshold $\delta$ is also applied here.

The order-vector-based approach has the following advantages. Primely, the evaluation order vector is a more robust and reliable indicator to detect the selectivity change, because it reflects the intrinsic priority of filter evaluation. Secondly, we define the judgment criterion based on the PCA in high-dimensional space, which can stably reflect the overall distribution of the order vectors and reduce the detection complexity. Thirdly, the threshold of judgment is derived from normalized cosine similarity measure, which is more general and reliable, and highly efficient to evaluate.

\subsection{Selectivity Updating}

In this part, we will describe the specific approach to update the selectivity estimation for each filter if we have detected the overall change in selectivity distribution. Our approach is based on the statistical estimation but will emphasize the balance of overhead in selectivity updating.

To estimate the new selectivity value of a filter, we propose to make use of its existing actual evaluation outcomes. Evidently, we can employ " 1 " and " 0 " to represent the evaluation outcomes "True" and "False" respectively. With the assumption that the data items are mutually independent, the evaluation results can be modeled as following a Bernoulli distribution. By this means, the filter selectivity can be estimated by the binomial proportion of the results evaluated as "1" ("True"), which is,

$$
\hat{s}=\frac{\sum_{t} o_{(t)}}{L_{o}} .
$$




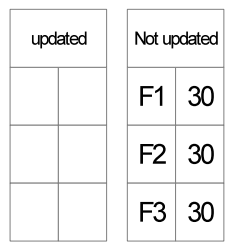

(a) Initialization

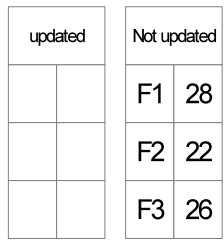

(b) In adaption

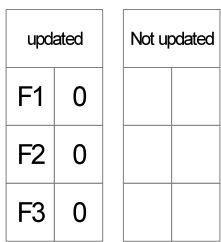

(c) Adaption completed
Fig. 4. Filter maintenance of FilAda for filter adaption.

Here, $o_{(t)}$ is the evaluation outcome at time stamp $t$ and $L_{o}$ is the total number of observations.

For each filter, we need sufficient observations to estimate the selectivity. Because we only detect the variation of overall selectivity distribution, all filters' selectivity values have to be reestimated. Intuitively, from the point of confirming the selectivity variation, we can process the following data items with all filters fully evaluated and record the evaluation outcomes. When the sample size is sufficient, we can recalculate each of the new selectivity value. However, such naive approach will introduce too much overhead to process each data item group and is unacceptable in practical processing.

To overcome the drawbacks above, we propose a dynamical weighted evaluation strategy to update selectivity with restricted overhead. This strategy dynamically obtains filter evaluation samples during processing data items, while providing fair chance to each filter by weighted selection, so as to balance their sample sizes. More specifically, to begin the updating, all filters will be marked as "not updated" and assigned with the same initial weight ${ }^{5} 30$ and sample size 0 . To execute a new data item group, the filters will be randomly and dynamically selected based on their weights to answer the query set, and a high weight means a high probability to select such a filter. If a filter is evaluated, its weight will be reduced by one, and the sample size will increase by one; the filters remained in the residual filter-query graph will be further selected based on their weights. In the updating process, the weight will be always inversely proportional to the filter's present sample size. When a filter's weight becomes zero, it means we have sufficient samples and its selectivity estimation can be updated immediately. Such filter will be marked as "updated", and its selection priority will be lower than all those not updated. The adaption procedure is completed when all filters are successfully updated. In this way, we are able to collect sufficient samples for each filter, and the overhead can be effectively controlled. After the updating, the framework will process and monitor the following data items with new selectivity estimations. Fig. 4 exemplifies an instance of filter maintenance in updating module.

\subsection{Discussions on FilAda}

The FilAda is a general and efficient framework for dynamic filter adaption, which follows the basic monitor-

5. We apply 30 because it is a statistically accepted number for sufficient sample size. and-update model. In the procedure of FilAda, we can conclude that the updating module will potentially spend more execution cost for sample collection and sacrifice certain efficiency, but the monitoring module is efficient and needs no extra cost. However, although FilAda works well when the overall selectivity distribution collectively change, the framework still has to face the problem that the filters are mutually independent and the filter selectivity may vary individually and asynchronously. If at a certain time stamp, the selectivity change only takes place to a small number of filters (e.g., one or two filters), the induced influence on the filter evaluation order may be limited and not lead to salient variation. However, the accumulative affection will show out gradually. In this situation, the proposed solution may fail to achieve the expected performance, and we need a more sophisticated approach to monitor the filter selectivity change and update it accordingly.

\section{AdVANCED APPROACH FOR FILTER ADAPTION}

To address the limitation of FilAda, we propose an advanced strategy named A-FilAda which is able to detect asynchronous selectivity variation.

\subsection{Discussion on Single Filter}

At first, we discuss the monitoring and selectivity updating of a single filter. Following the process of hypothesis testing, we estimate the filter selectivity for current streaming data, and then compare it with the pre-known value to judge whether the selectivity needs updating.

As described in the previous section, the filter evaluation results can be modeled as following a Bernoulli distribution. When we have recorded the evaluation outcomes of a filter in the sliding window, we can immediately estimate its selectivity by Equation (4). To determine whether the selectivity needs updating, we accept estimation error and apply confidence interval to provide a range that contains the actual value with certain confidence, so we need to check whether the pre-known value falls within the range.

There are various approaches to calculate the confidence interval for a binomial proportion, and the most widely used one is normal approximation interval, which assumes that the estimation error follows a normal distribution. In such an assumption, the confidence interval can be calculated as $\left[\hat{s}-z \sqrt{\frac{1}{L_{o}} \hat{s}(1-\hat{s})}, \hat{s}+z \sqrt{\frac{1}{L_{o}} \hat{s}(1-\hat{s})}\right]$, where $z$ is the $1-\frac{1}{2} \alpha$ percentile of normal distribution ( $\alpha$ is the error percentile). Usually, we choose the confidence level at $95 \%$, and the corresponding percentile $z=1.96$. However, it is found that the confidence interval based on normal approximation has unsatisfactory performance when $\hat{s}$ is close to 0 or 1 , while in practice, the filter selectivity is usually close to extreme probability. To tackle this, the confidence interval based on Wilson score [31] has been proposed, and the interval range is modified to

$$
\frac{1}{1+\frac{1}{L_{o}} z^{2}}\left[\hat{s}+\frac{1}{2 L_{o}} z^{2} \pm z \sqrt{\frac{1}{L_{o}} \hat{s}(1-\hat{s})+\frac{1}{4 L_{o}^{2}} z^{2}}\right] .
$$


In our work, we accept the advanced Wilson score and refine the interval calculation by Wilson interval, which is more suitable for our problem scenario.

Giving such a confidence interval, if the pre-known selectivity $s$ is within the interval, we can claim that the selectivity still holds with $95 \%$ confidence and keep it unchanged. Otherwise, the hypothesis is rejected and we can replace $s$ with $\hat{s}$ immediately.

\subsection{Monitoring Multiple Filters}

Now we interpret how to deal with multiple filters based on the sliding window information. We cannot simply extend the single filter case to multiple filters, because in the multi-filter scenario, when considering a snapshot of the sliding window, the sample sizes of filter evaluation results vary much among different filters, since not every filter is evaluated at each time stamp. As a result, some filters' sample sizes may remain at a low level. Evidently, we expect enough samples to enable a more statistically reliable estimation of selectivity. Therefore, our approach needs to achieve the following objectives: 1 . Continuously estimating and updating the selectivity of those filters with sufficient sample sizes; 2. Dynamically increasing additional observations for those filters with low sample sizes. To increase the filter sample size, one approach is to spend extra cost to test more filters after regular query execution. However, for the efficiency of stream processing, the cost quota is not unlimited. Therefore, how to distribute the extra cost is an important issue to resolve. Next, we will interpret and emphasize the extra filter sampling and the cost management.

\subsubsection{Filter Selection Probability for Extra Sampling}

Firstly, according to the observation number of each filter in the sliding window, we divide the filters into two categories, namely, lowFilter and highFilter, to represent the filters with low and high sample size respectively. We still select sample size 30 as the default threshold.

We aim to perform extra sampling on filters in lowFilter after regular query execution. To make the best use of extra cost, we are motivated to select those most influential filters in lowFilter to further verify. According to the graph model, the number of edges that a filter can cover plays an important role in filter ordering. Therefore we propose to measure a filter's influence based on its edge covering. As defined in preliminaries, the edge number that one filter covers is denoted as " $E^{T}$ " and " $E^{F}$ " when the filter is "True" and "False", respectively. Considering both evaluation outcomes, we calculate a weighted sum to estimate a filter's edge covering, i.e., $w_{1} * E^{T}+w_{2} * E^{F}$, where $w_{1}+w_{2}=1 . w_{1}$ and $w_{2}$ can be adjusted to emphasize one evaluation result, and we set $w_{1}=w_{2}=0.5$ by default. In addition, we combine the filter's current selectivity to form the influence measure. That is, for $F_{i}$,

$$
I_{i}=\left(w_{1} * E^{T}+w_{2} * E^{F}\right) * s_{i} .
$$

The influence measure is proportional to the selectivity, because if a filter is likely to be "False" but still with low sample size, it can probably affect few edges; on the contrary, those filters with high probability to be "True" can potentially show more influence if its selectivity changes.

By this means, we can spend the extra cost on those filters top-ranked by the influence score. Nevertheless, for the sake of fairness, it is also necessary to consider the filters with relatively lower influence. We propose to select the filters according to certain probability distribution, and those more influential filters are assigned with higher probability so that they have more chance to be extra sampled. Naturally, according to the influence measure, the selection probability for filter $F_{i}$ can be calculated as:

$$
p_{i}^{s}=\frac{I_{i}}{\sum_{F_{j} \in \text { low Filter }} I_{j}} .
$$

\subsubsection{Sampling Priority by Post-Examining}

The filter influence score can help to distribute extra cost for filter sampling, but lack the flexibility to the actual streaming data. We further propose a post-examining approach to assist the effective extra sampling, by using the actual evaluation results obtained by processing current data items. For example, assume that a filter's selectivity is 0.8 , and its evaluation result will be "True" with high probability. If the actual result is "False", its selectivity may have changed, and we can set priority to it for extra sampling. We propose to use Bayes' theorem to discuss how to employ this postexamining knowledge to improve extra cost distribution.

Bayes' theorem expresses posterior probability by the likelihood and prior probability. For a single filter, let $A$ represent the event that the selectivity of the filter is changed, and $B$ denote the event that the filter evaluation result is "True" 6 . According to the Bayes' theorem, given the fact that the filter outcome is "True", the probability of selectivity change is

$$
\begin{aligned}
P(A \mid B) & =\frac{P(B \mid A) P(A)}{P(B)} \\
& =\frac{P(B \mid A) P(A)}{P(B \mid A) P(A)+P(B \mid \neg A) P(\neg A)} .
\end{aligned}
$$

We notice that, if the filter selectivity changes, the probability of evaluation result being "True" $P(B \mid A)=0.5$, since the new selectivity value can be random in $[0,1]$. Assuming that the prior probability of selectivity change $P(A)=a$, the above equation can be derived into

$$
P(A \mid B)=\frac{\frac{1}{2} \cdot a}{\frac{1}{2} \cdot a+s \cdot(1-a)}=\frac{1}{1+\frac{2 s(1-a)}{a}} .
$$

By default, let $P(A)=a=0.5$, and we have ${ }^{7}$

$$
P(A \mid B)>0.5 \quad \Leftrightarrow \quad s<\frac{a}{2(1-a)}=0.5 .
$$

6. The case that the filter outcome is "False" is symmetrical to the "True" case. All discussions are directly applicable.

7. If we have prior information about the stream to determine the probability of filter selectivity change, we can set different value to $P(A)$. Generally, $a \leq 0.5$ because the stream is relatively stable and selectivity change will not usually take place. 
It means if the pre-known selectivity $s$ is less than 0.5 , we can suspect the selectivity change when the result is "True". The general rule is, if the actual evaluation result is contradictory to the expectation, we can suspect the potential selectivity change.

Based on the above analysis, our extra cost distribution strategy can be modified to make use of the actual evaluation results. If the filter evaluation result indicates the high suspicion of selectivity change, we can assign priority to extra evaluate such a filter when processing the next data item, so as to guarantee its evaluation after the regular query execution. Otherwise, we remove its priority, and it will be selected according to the selection probability $p_{i}^{s}$.

More formally, whether we should assign selection priority depends on the relationship between a filter's expected result, i.e., pre-known selectivity $s_{i}$, and its actual evaluation result $o_{i}$. We denote the selection priority of filter $F_{i}$ by $p_{i}^{r}$. When the pre-known selectivity of a filter is less than 0.5 , if its actual evaluation result is "True", we claim a suspicion and assign evaluation priority to it for next data item $\left(p_{i}^{r}=1\right)$, otherwise we remove the priority $\left(p_{i}^{r}=0\right)$. The situation is similar for the case that the pre-known selectivity is greater than 0.5 . By defining a sign function

$$
\operatorname{sgn}(x) \triangleq \begin{cases}0 & x \leq 0 \\ 1 & x>0\end{cases}
$$

we have

$$
p_{i}^{r}=\operatorname{sgn}\left[\left(0.5-s_{i}\right)\left(o_{i}-0.5\right)\right]
$$

Sometimes, we may need to control the criterion of priority assignment, e.g., 0.5 may be too loose to detect the abnormal evaluation result, since with $50 \%$ probability, the chance of getting "True" and "False" results are equal. Therefore, we need to set a higher criterion, e.g., only the evaluation results conflicting with more extreme selectivity will be considered as abnormal. We still use the above example to explain. Let $p_{c}$ be a more extreme selectivity, we have

$$
s<p_{c}<0.5 \Leftrightarrow P(A \mid B)>\frac{1}{1+2 p_{c}}>0.5 .
$$

Here $p_{c}$ acts as a factor controlling the criterion of priority assignment, e.g., if $p_{c}=0.3$, we will only consider the preknown selectivity less than 0.3 , which is a more extreme probability and can be more reliable. Similarly, the other extreme situation that $p_{c}>0.5$ is also applied.

In this way, we are able to spend the extra cost as necessary as possible, and the extra filter selection can be dynamically adjusted to be adaptive to the actual data items. Evidently, we can further improve the suspicion by checking several consecutive evaluation outcomes, but for the simplicity, we only consider the current result. Such a selectivity adaption is able to benefit the overall optimization of the continuous multiple query execution and potentially reduce the processing cost in average.

\subsection{Mechanism of A-FilAda}

Summarizing the above discussions, in this part, we formally provide the systematic description of A-FilAda for

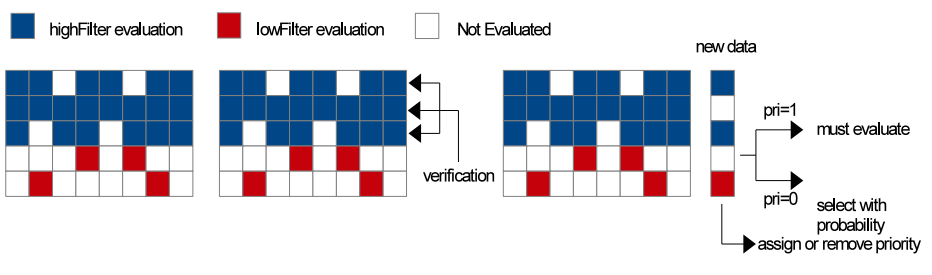

(a) Initialization

(b) highFilter verification

(c) lowFilter extra sampling

Fig. 5. Mechanism of A-FilAda.

dynamic filter adaption, and interpret each procedure in details. The overall framework is interpreted in Fig. 5.

Initialization: Firstly, an initialization procedure is proposed to start the stream processing (Fig. 5 (a)). Here we assume that the initial selectivity value for each filter is pre-known. Naturally, we start by using these priori knowledge and completing the initial information of the sliding window statistics. We apply the graph-based approach to execute the queries for each group of incoming data items, and for each filter, record whether it is evaluated and the actual evaluation result. The initialization is completed when the sliding window is fully filled.

Filter Categorization: Before processing new data items, for each filter, we count its observation number in the window. Based on this, we categorize the filters into highFilter and lowFilter for following processing. The categorization can be incrementally updated after the initialization.

HighFilter Verification: After the identification of highFilter and lowFilter sets, we can instantly verify the filters of highFilter, and check whether their selectivity values are influenced according to binomial proportion testing based on Wilson interval. If any changes of selectivity are detected, we update the corresponding values immediately.

Regular Processing: After verifying those filters with sufficient samples, we start regular query execution to process the current data item group, using the graph-based approach. After that, we can divide the filters of lowFilter set and mark them as "evaluated" and "unevaluated" for following extra evaluation.

LowFilter Sampling Processing: As described in Fig. 5 (c), we spend extra cost to increase the evaluation samples of the filters in lowFilter. For those evaluated filters, we compare the evaluation results with the expectation and decide whether to assign them evaluation priority for future sampling process. For those unevaluated filters, we first check whether they have been assigned evaluation priority. If positive, we evaluate the filter and judge again whether the priority should be remained or removed; otherwise, we select some of them to make extra evaluation based on the probability distribution of filter selection (Equation (6)). Whenever the filter results are available, we can adjust their sampling priorities.

Window Updating: After processing the current data items, we incrementally update the window statistics, and then loop from the Filter Categorization procedure until the data stream ends. 


\section{EXPERIMENTS}

In this section, we will present the empirical evaluation of our approaches and illustrate their performance in improving the cost management. We conduct the experiments on a range of synthetic and real data, and compare the results of our approaches with those achieved by other methods [5].

Since we focus on the optimization of cost management, the actual execution cost spent on each data item is employed to measure and evaluate the performance of different approaches. For synthetic data, we define the virtual cost value for each filter, so the execution cost is the sum cost of all evaluated filters; while for real data, we use the execution time to quantify the cost.

In the empirical study, firstly, we will describe the datasets in use, especially the generation of synthetic data. Next we examine the general performance of FilAda and A-FilAda solutions by a range of parameter tuning on synthetic data. The cost results will be compared with those achieved by other strategies, which all follow the filter ordering based on Residual Graph [5] but with different filter adaption strategies. The first one always has in advance the ground truth information, i.e., the actual selectivity values, so filter adaption is not needed (denoted as "GT"); the second one takes no filter adaption (denoted as "NA"); the third one adopts periodic filter adaption and performs updating every 500 samples, with the updating approach same as FilAda (denoted as "Periodic"). After the general comparison, we will further study trade-off issues and provide a detailed comparison of FilAda and A-FilAda to discuss their advantages and disadvantages. Finally, we will demonstrate the framework performance on two real data sets, the traffic and multimedia data.

\subsection{Dataset Description}

\subsubsection{Synthetic Data Simulation}

To generate the synthetic data, we follow the work [4] and design an instance generator to establish the simulative filter-query relationship. To initialize an instance, the generator will create $n$ filters and specify each filter's cost, selectivity and popularity values, which are randomly chosen from prescribed value ranges. $m$ queries are defined on these filters by recording the filter assignment.

With each identified instance, we are able to generate $n$ data streams by simulating the consecutive data items. For each time stamp, the data group will be represented by the status of filter evaluation on them, i.e., an n-value vector recording the evaluation results of each filter, simulated by randomly deciding whether each filter $F_{i}$ is "True" with the probability $s_{i}$. To denote the stream variation, we can manually change the filter selectivity distribution during data stream processing, and control the proportion of affected filters. To emphasize the selectivity change, there will be salient difference between new value and the original one, i.e., $s_{i}^{\prime}=\left(s_{i}>0.5\right) ?\left(s_{i}-0.5\right):\left(s_{i}+0.5\right)$. The default setting of the parameters are listed in Table 2, which can be tuned to stimulate different conditions.
TABLE 2

\section{Parameter setting}

\begin{tabular}{|c|c|}
\hline Parameter & Default setting \\
\hline Number of filters & 100 \\
\hline Number of queries & 150 \\
\hline Range of cost values & {$[8,10]$} \\
\hline Range of popularity values & {$[20,30]$} \\
\hline Range of selectivity values & {$[0,0.25]$} \\
\hline Window length & 200 \\
\hline Proportion of affected filters & $30 \%$ \\
\hline Periodic checking frequency & 500 \\
\hline
\end{tabular}

\subsubsection{Real Data Description}

A traffic dataset and a multimedia dataset are collected for real data evaluation. The traffic dataset is provided by the CityPulse EU FP7 Project [32]. It is a collection of vehicle traffic in cities of Denmark. Each stream records the traffic status between two points over a period of 6 months (449 data streams in total). The observations include the average vehicle speed, the vehicle count, etc., and our filters are defined based on these observations.

For multimedia data, we randomly select $25 \mathrm{TV}$ commercials and connect them to form a long video stream. Each commercial is 1 minute long with frame rate at 25 fps, and the resolution of single frame is about $370 * 250$. The filters are defined on the frame images to detect certain image feature, e.g., to determine whether the proportion of pixels in a specific color is higher than a threshold. These filters are combined to form different queries on the frames.

\subsection{Evaluation by Synthetic Data}

\subsubsection{Effect of Variation Synchrony}

Firstly, we examine the performance of different approaches on the streams with synchronous and asynchronous variation respectively, so as to explore the effect of variation synchrony, especially on our proposed approaches. We compare all strategies with default parameter settings, and record the average query execution cost considering both in and after stream variation ${ }^{8}$. The results are shown in Fig. 6.

From the figure we can discover that, the FilAda and AFilAda can both improve the query execution by filter adaption procedure, but for different kinds of stream variation, they will have different performance. Generally speaking, if the selectivity change collectively, FilAda can precisely detect such variation and update the filter effectively, but for asynchronous variation, A-FilAda can perform better since it deals with the filters individually. For the following empirical studies on synthetic data, to simulate more general situation, we will deliver the experiments on streams with asynchronous variation if not specified otherwise.

\subsubsection{Effect of Popularity Range}

Next, we focus on the effect of filter's popularity range. The popularity denotes the sharing degree of each filter,

8. We select equal number of records in variation stage and after variation stage to calculate the average cost for fairness. 


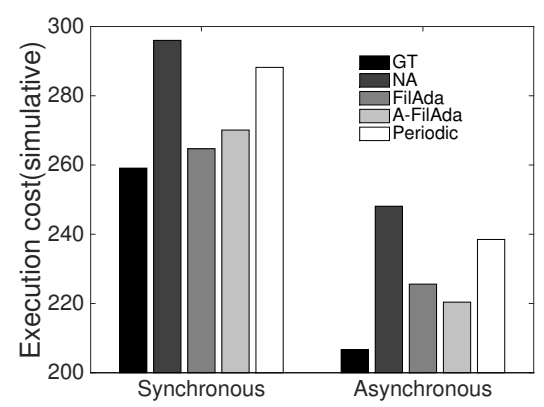

Fig. 6. Effect of stream variation synchrony.

so different value range will affect the filters' significance in query execution. In this study, we tune the popularity range as $[10,20],[20,30],[30,40]$ and $[40,50]$, i.e., $10 \%$ to $30 \%$ sharing degree. For each range, we generate the problem instances, create corresponding data streams, and run all strategies on the streams with simulated changing selectivity. For each strategy, the average execution costs on varying and stable data are recorded, and Fig. 7 denotes the trend as popularity range increases.

The figure shows that the overall execution cost will drop as the popularity increases, because the filter can potentially answer more queries with larger sharing degree. A-FilAda can outperform other strategies and reduce $5 \%$ to $10 \%$ cost. It should be notice that FilAda and periodic checking cannot effectively reduce the average cost, because the streams perform asynchronously variation, so they have to carry out several times of filter updating procedures, which will increase the average cost.

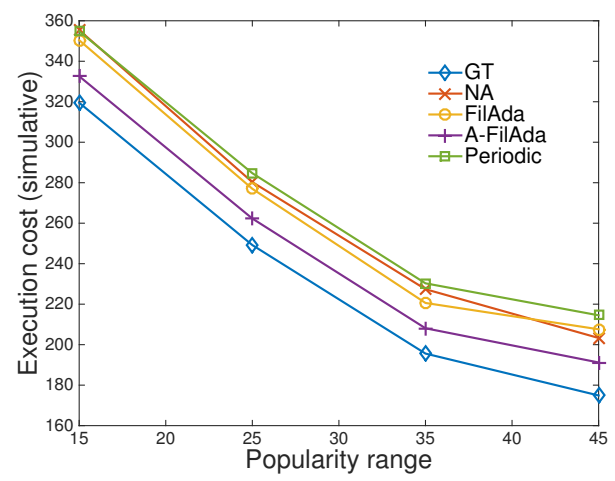

Fig. 7. Effect of filter popularity range.

\subsubsection{Effect of Selectivity Range}

Then we examine the effect of filter's selectivity range. In our instance setting, the initial selectivity range will determine the new range after variation, which will also affect the overall query execution. In this study, we follow the stream generation strategy above, and tune the selectivity range as $[0,0.25],[0.25,0.5],[0.5,0.75]$ and $[0.75,1]$. The corresponding average execution costs of different strategies in stream processing are recorded in Fig. 8.

The figure curve shows an interesting trend due to the initial selectivity range and the way we simulate the

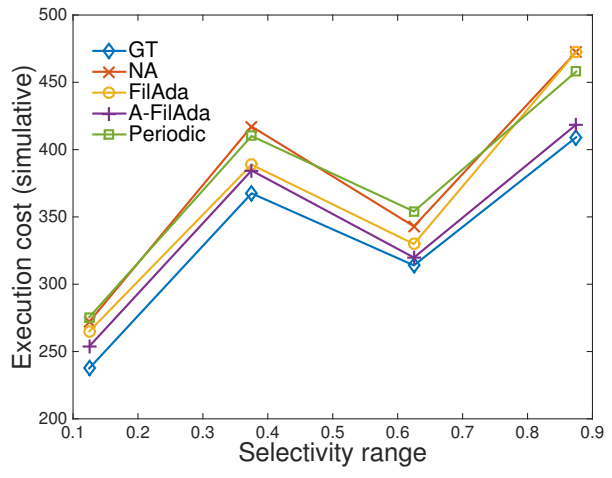

Fig. 8. Effect of filter selectivity range.

selectivity change. With extremely small selectivity range, the query set can be answered by relatively small number of filters, even if $50 \%$ filters change their selectivity value. For the cases that selectivity range is close to 0.5 , the overall execution cost will be higher, but for the initial range of $[0.5,0.75]$, the varied selectivity will fall into $[0,0.25]$, so the execution cost will be reduced after filter adaption, which results in the slightly less average cost. As to the case of initial range $[0.75,1]$, the situation is opposite to the case of $[0,0.25]$, so the average cost is much higher than other cases. We can discover that A-FilAda approach can outperform other competitors by up to $15 \%$ cost reducing, and when the initial selectivity range falls in $[0.75,1]$, AFilAda achieves most cost saving.

\subsubsection{Effect of Proportion of Affected Filter}

Finally, we focus on the effect brought by the proportion of affected filters in stream variation. This proportion will reflect the overall change in stream status, and thus show great influence on the performance of our framework. In this study, we control the proportion of streams with salient variation from 0.3 to 0.9 , and also record the average execution cost during stream processing of different strategies. Fig. 9 denotes the cost trend following different proportion.

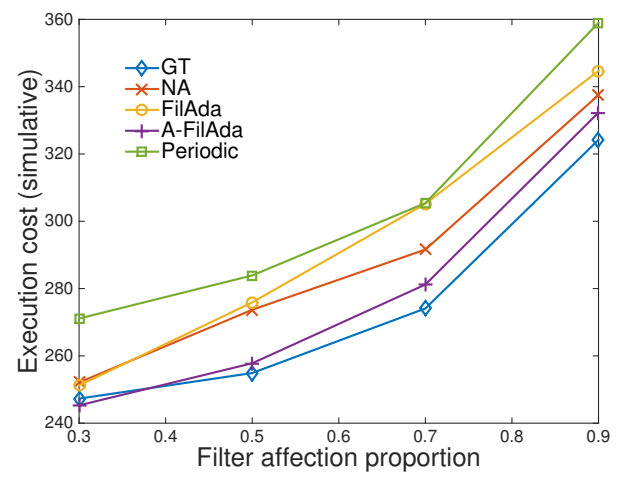

Fig. 9. Effect of proportion of filter affection.

The overall execution cost will increase as the proportion of varied streams increases, because if more filters' selectivity change to larger values, the query set will be 
TABLE 3

PCA VS. Reference-Vector in FilAda.

\begin{tabular}{|c|c|c|c|c|c|c|c|}
\hline \multirow{2}{*}{ rangeS } & \multirow{2}{*}{ propC } & \multicolumn{2}{|c|}{ PCA200 } & \multicolumn{2}{c|}{ PCA160 } & \multicolumn{2}{c|}{ RV160 } \\
\cline { 2 - 8 } & & FA & FD & FA & FD & FA & FD \\
\hline \multirow{5}{*}[0,0.3]{} & 0.05 & 2 & 0 & 2 & 1 & 0 & 1 \\
\cline { 2 - 8 } & 0.1 & 1 & 0 & 3 & 0 & 0 & 1 \\
\cline { 2 - 8 } & 0.15 & 3 & 0 & 0 & 1 & 0 & 1 \\
\cline { 2 - 8 } & 0.3 & 1 & 0 & 2 & 0 & 0 & 0 \\
\cline { 2 - 8 } & 0.5 & 2 & 0 & 5 & 0 & 0 & 0 \\
\cline { 2 - 8 } & 0.7 & 1 & 0 & 3 & 1 & 0 & 0 \\
\hline \multirow{5}{*}[0.7,1]{} & 0.05 & 0 & 1 & 0 & 1 & 0 & 1 \\
\cline { 2 - 8 } & 0.1 & 0 & 1 & 0 & 1 & 0 & 1 \\
\cline { 2 - 8 } & 0.15 & 0 & 1 & 0 & 1 & 0 & 1 \\
\cline { 2 - 8 } & 0.3 & 0 & 0 & 0 & 1 & 0 & 0 \\
\cline { 2 - 8 } & 0.5 & 2 & 0 & 3 & 0 & 0 & 0 \\
\cline { 2 - 8 } & 0.7 & 2 & 0 & 3 & 0 & 0 & 0 \\
\hline
\end{tabular}

potentially answered by more filters. A-FilAda still shows best performance among all competitors with around $10 \%$ cost saving. It should be noticed that the cost of FilAda will gradually surpass the baseline NA, because as the proportion of filter affection increases, FilAda has to carry out more times of filter updating to make sure all filters are updated, which will bring much burden and increase the average cost.

\subsubsection{Comparison of Different Solutions in FilAda}

After the general evaluation, we further discuss the potential solutions applied in FilAda, and examine the influence of window size in filter adaption. We compare the performance of PCA and the reference-vector (RV) approaches under different conditions, and the results are listed in Table 3. Here, "rangS" denotes the selectivity range of each filter; "propC" means the proportion of affected filters in stream variation; "FA" means false alarm and "FD" means false dismissal. "PCA200" means the window size is 200 and PCA is applied for filter adaption, so for "PCA160", the window size is insufficient since there are 100 filters.

As indicated, there will be false alarms for PCA approach, and the performance becomes worse when the window size is insufficient, because it will cause the instability of PCA analysis. RV approach can achieve satisfactory performance with different window sizes, but there will be unavoidable false dismissals when the proportion of affected filters is small. With small proportion of filter affection, when the selectivity range is high, false dismissals occur to both solutions. In general, PCA approach is more sensitive and brings in more false alarms, while RV approach cannot well handle small affection proportion and may make false dismissals which are more severe mistakes.

\subsubsection{Stability Test on Variation Frequency}

To examine the stability of our approaches in various scenarios, we evaluate on data streams with different frequencies in selectivity variation. In the stream stimulation, we set the stream variation frequency as $1 / \kappa$ (selectivity changes every $\kappa$ samples), e.g., $\kappa=50,100,200,400,800$.
The average query execution costs of different approaches at different variation frequency are shown in Fig. $10^{9}$.

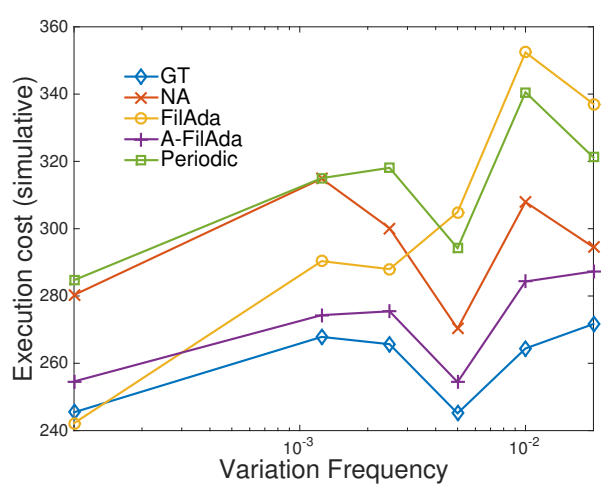

Fig. 10. Effect of variation frequency.

The figure shows that A-FilAda can effectively reduce the execution cost for streams with different variation frequencies. FilAda behaves even better than A-FilAda when the stream is relatively stable (with only one change), due to the insignificant cost of filter adaption. FilAda however loses the superiority when the stream changes rapidly.

\subsubsection{Trade-off Issues Discussion}

This part will focus on trade-off issues in cost management, including the effective distribution of extra cost for filter adaption, and the difference of overhead between FilAda and A-FilAda strategies. We will discuss the details by empirical study.

Issue 1:The objective of filter adaption is to eventually reduce the query execution cost, while the selectivity updating will spend extra cost for filter sampling. Therefore, there is a trade-off between the extra cost spent in filter adaption and the benefit in cost reduction for overall query execution, so it is essential to control the extra cost in filter adaption. To examine this trade-off, we record the average execution cost of our solutions in and after filter adaption procedure respectively. The results in these two stages are compared with the those achieved without filter adaption, as shown in Table 4 and Table 5, for FilAda and A-FilAda, respectively. In the table, "V" and "S" stand for "Varying" and "Stable", i.e., in and after filter adaption. We are interested in both absolute and relative difference, and "Comparison" column records the relative pay of the corresponding solution over "NA" in "V" stage and relative gain over "NA" in "S" stage, i.e., for "V" stage, Comparison $=\frac{\text { Solution }-\mathrm{NA}}{\mathrm{NA}} \times 100 \%$, and for "S" stage, Comparison $=\frac{\text { NA-Solution }}{\text { NA }} \times 100 \%$.

From the tables, we can conclude that A-FilAda can generally pay less cost to gain even more cost saving in stream stable stage, and the benefit amount increases as more filters have changed the selectivity. The only exception is for the $10 \%$ change proportion, in which the pay will be more than cost gain, because the number of affected filters is small, so the overall filter evaluation is not saliently influenced. The pay and gain relationship is

9. The leftmost results are from streams with only one change. 
TABLE 4

Trade-off between pay and gain in FilAda.

\begin{tabular}{|c|c|c|c|c|c|}
\hline rangeS & propC & Stg & FilAda & NA & Comparison(\%) \\
\hline \multirow{8}{*}[0,0.3]{} & \multirow{2}{*}{0.1} & V & 338.0673 & 225.9827 & 49.60 \\
\hline & & $\mathrm{S}$ & 226.3609 & 228.8322 & 1.08 \\
\hline & \multirow{2}{*}{0.3} & V & 352.4829 & 295.1958 & 19.41 \\
\hline & & $\mathrm{S}$ & 213.7182 & 297.5501 & 28.17 \\
\hline & \multirow{2}{*}{0.5} & $\mathrm{~V}$ & 403.2507 & 318.0849 & 26.77 \\
\hline & & $\mathrm{S}$ & 236.4005 & 307.1372 & 23.03 \\
\hline & \multirow{2}{*}{0.7} & V & 582.9557 & 504.323 & 15.59 \\
\hline & & $\mathrm{S}$ & 385.3052 & 513.8457 & 25.02 \\
\hline \multirow{8}{*}[0.7,1]{} & \multirow{2}{*}{0.1} & $\mathrm{~V}$ & 673.1896 & 641.2726 & 4.98 \\
\hline & & $\mathrm{S}$ & 625.0029 & 650.3749 & 3.90 \\
\hline & \multirow{2}{*}{0.3} & $\mathrm{~V}$ & 563.2366 & 493.0586 & 14.23 \\
\hline & & $\bar{S}$ & 380.4966 & 481.3159 & 20.95 \\
\hline & \multirow{2}{*}{0.5} & V & 412.6117 & 348.513 & 18.39 \\
\hline & & $\mathrm{S}$ & 266.7872 & 349.9009 & 23.75 \\
\hline & \multirow{2}{*}{0.7} & V & 384.8884 & 304.0767 & 26.58 \\
\hline & & $\bar{S}$ & 252.7262 & 306.702 & 17.60 \\
\hline
\end{tabular}

TABLE 5

Trade-off between pay and gain in A-FilAda.

\begin{tabular}{|c|c|c|c|c|c|}
\hline rangeS & propC & Stg & A-FilAda & NA & Comparison(\%) \\
\hline \multirow{8}{*}[0,0.3]{} & \multirow{2}{*}{0.1} & $\mathrm{~V}$ & 226.8892 & 222.7846 & 1.84 \\
\hline & & $\mathrm{S}$ & 221.1351 & 224.8084 & 1.63 \\
\hline & \multirow{2}{*}{0.3} & V & 262.232 & 267.0144 & -1.79 \\
\hline & & $\mathrm{S}$ & 254.845 & 300.4355 & 15.17 \\
\hline & \multirow{2}{*}{0.5} & $\mathrm{~V}$ & 276.1453 & 310.6116 & -11.10 \\
\hline & & $\mathrm{S}$ & 301.0243 & 389.5963 & 22.73 \\
\hline & \multirow{2}{*}{0.7} & $\mathrm{~V}$ & 305.1697 & 360.5866 & -15.37 \\
\hline & & $\mathrm{S}$ & 366.1061 & 501.6188 & 27.02 \\
\hline \multirow{8}{*}[0.7,1]{} & \multirow{2}{*}{0.1} & $\mathrm{~V}$ & 627.959 & 632.7669 & -0.76 \\
\hline & & $\mathrm{S}$ & 619.0438 & 626.1252 & 1.13 \\
\hline & \multirow{2}{*}{0.3} & $\mathrm{~V}$ & 499.2116 & 530.9495 & $\begin{array}{l}-5.98 \\
\end{array}$ \\
\hline & & $\mathrm{S}$ & 384.9868 & 448.378 & 14.14 \\
\hline & \multirow{2}{*}{0.5} & $\mathrm{~V}$ & 423.1145 & 453.5657 & -6.71 \\
\hline & & $\mathrm{S}$ & 293.3235 & 333.0124 & 11.92 \\
\hline & \multirow{2}{*}{0.7} & V & 384.6511 & 404.9286 & -5.01 \\
\hline & & $\mathrm{S}$ & 243.2799 & 263.8183 & 7.79 \\
\hline
\end{tabular}

more obvious for FilAda. In Varying stage, FilAda will pay about $20 \%$ more cost for filter adaption, and such number can go up to $49 \%$ in some cases, while the gain in stable stage is also around $20 \%$. There is no obvious rule about when the pay will surpass the gain and when not, but when the proportion of affected filters is small, the benefit is not impressive. Therefore, we recommend that the framework can achieve best performance when the variation occurs among large proportion of filters.

Issue 2:Another issue to discuss is the difference between FilAda and A-FilAda strategies. The A-FilAda strategy spends the extra cost for filter adaption in addition to the regular query processing, which means the extra cost is spent averagely for each set of data items. The FilAda strategy is more suitable for the case in which the selectivity change at the same time, and there will be no extra cost in regular processing due to the efficient monitoring module, while relatively high cost will be centralized during the overall selectivity updating. In this respect, although AFilAda strategy is more sophisticated to deal with different situations, it will potentially introduce unnecessary cost in
TABLE 6

Comparison on traffic streams

\begin{tabular}{|c|c|c|c|}
\hline Popularity Range & A-FilAda & FilAda & NA \\
\hline$[0,10]$ & 51.11 & 59.81 & 59.45 \\
\hline$[10,20]$ & 21.94 & 31.76 & 31.29 \\
\hline$[20,30]$ & 14.80 & 22.62 & 25.98 \\
\hline$[30,40]$ & 11.25 & 17.54 & 22.78 \\
\hline
\end{tabular}

regular processing. Thus, the different cost distribution is also a point we are interested in.

We compare FilAda and A-FilAda in details and study their cost in different stages. We use "Initial", "Varing" and "Stable" to represent the stages before, in and after stream variation. For fair comparison, we conduct FilAda and AFilAda on the data streams with synchronous variation. We compare the performance of two solutions in Varying and Stable stages, with the baseline results achieved by "GT" in terms of relative cost value. The results are depicted in Fig. 11 and Fig. 12 for different selectivity ranges.

Generally, A-FilAda will spend around 3\% more cost in Stable stage, and the number can go up to $9 \%$ in a few cases. In Varying stage, the extra cost will only lie within $36 \%$. The FilAda solution, however, usually spends more than $50 \%$ extra cost in filter adaption, and the peak value can be $90 \%$. In return, the cost in Stable stage can be close to "GT" results, sometimes even less. Therefore, if we need to control the cost for all stages, A-FilAda is a better choice, and FilAda is suitable for reducing the cost in regular execution.

\subsection{Evaluation by Real Data}

In this part, we report the evaluation results of our solutions on real data. All algorithms are implemented by Matlab and the experiments are conducted by a PC running OSX system, with $2.5 \mathrm{GHz}$ CPU and 4GB memory.

We first test the performance of different approaches on the traffic data. Each traffic stream is assigned a filter and 150 queries are defined on these filters with varied popularity ranges. We record the average number of filters to answer the query set by FilAda and A-FilAda, and compare the results with the strategy without filter adaption (denoted as "NA"), which are shown in Table 6. Note that there is no ground truth as baseline in real applications.

The table indicates that the A-FilAda approach can effectively reduce the number of filter evaluation to answer the query set, and can outperform "NA" approach by up to $50 \%$. However, FilAda approach cannot effectively improve the performance over "NA", because the traffic status of different locations behave differently, and the stream variation will take place asynchronously, which will increase the frequency of selectivity updating for FilAda. When the popularity range increases from $[0,10]$ to $[30,40]$, the average cost will natually drop because each filter can potentially resolve more queries with larger sharing degree.

Then we focus on the video data. As previously mentioned, 25 video clips are connected to form the data stream. In our experiment, among every five frames we sample one to evaluate the query set, so for each video clip we can 


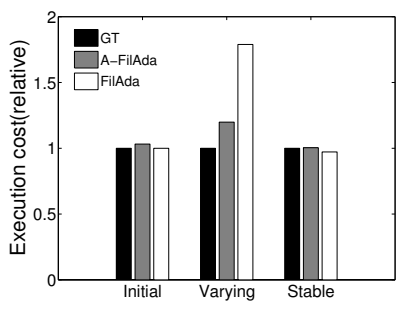

(a) Change proportion $=0.3$

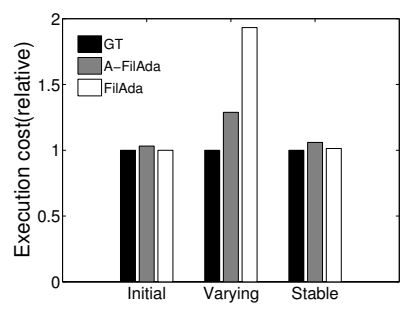

(b) Change proportion $=0.5$

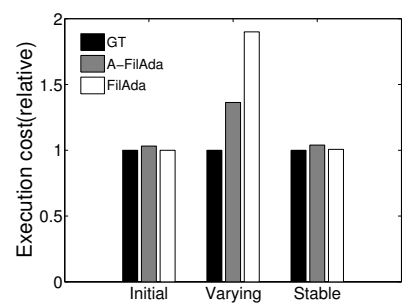

(c) Change proportion $=0.7$

Fig. 11. Detailed comparison between FilAda and A-FilAda: selectivity range $[0,0.3]$.

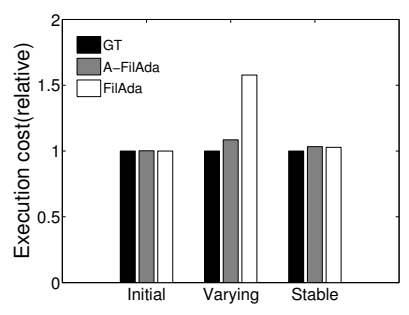

(a) Change proportion $=0.3$

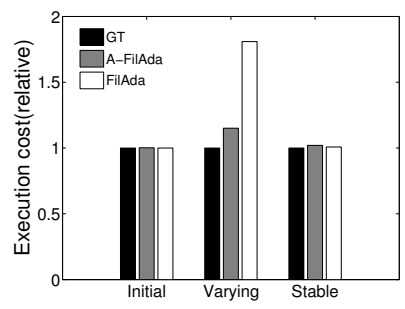

(b) Change proportion $=0.5$

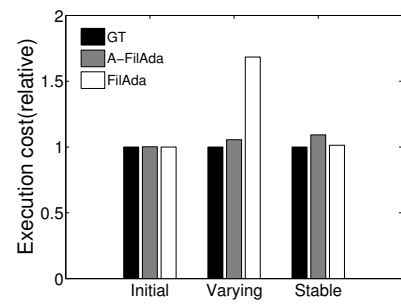

(c) Change proportion $=0.7$

Fig. 12. Detailed comparison between FilAda and A-FilAda: selectivity range $[0.7,1]$.

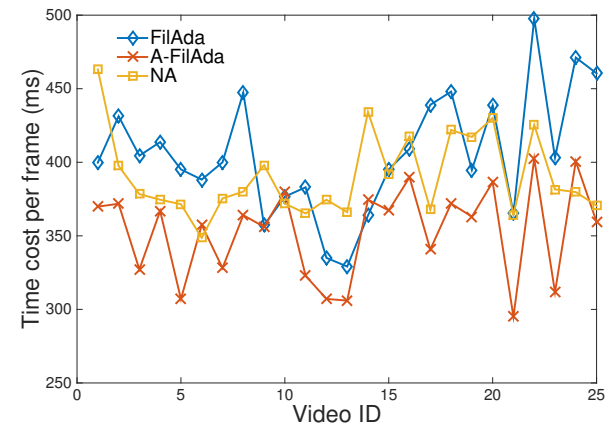

Fig. 13. Comparison on video stream.

have about 300 frames. In our experiment, we maintain 30 different filters and 50 queries with each filter shared among 6 to 10 queries. For each clip, we record the average time cost to process one frame and also compare three strategies. The comparison results are denoted in Fig. 13.

From the figure, we can discover that the A-FilAda can always restrict the overall time cost at a relatively low level, due to the ability of self adaption to the actual data characteristics. However, it cannot always guarantee the minimal results, because we set no limit to the cost amount to extra sampling, which may take relatively high proportion in overall cost. The time costs by FilAda solution show more salient fluctuation among different video clips, and even surpass the baseline "NA", because the TV commercials always contain rapid and frequent scene changing, which will result in frequent filter adaption and increase the average time cost to process the frames. More interesting is, if no filter adaption is taken, the average time cost is sometimes even lower than other approaches (for video 6 and 24). The reason is, in this application, most filters' selectivity will be close to zero, and only those matching the hue style of the initial video content can have higher selectivity values. Therefore, even if the video content has changed, sometimes the query set can be still solved directly by the first few filters in order, while the extra cost in filter adaption can be saved. Overall, AFilAda approach can effectively reduce the query execution cost by filter adaption.

\section{Conclusions ANd Future WORK}

This work addresses the challenges of cost management in optimizing multiple overlapping queries, where each query is a conjunction of expensive filters monitoring data streams and the filters can be shared among queries. Based on the initial study on optimal filter evaluation planning for stable data distribution, we further analyze the challenge of practical applications, in which model the data characteristics can potentially vary. Considering the influence of data fluctuation on the filter selectivity, a general framework is proposed in this work to achieve dynamic filter adaption, including selectivity monitoring and updating, which are independent to the actual data format. FilAda and A-FilAda are designed to address different scenarios and guarantee that filter ordering is adaptive to the actual data distribution. Extensive experiments on synthetic and real data indicate that our solutions can effectively reduce the processing cost to continuously execute the query set. The extra cost from filter adaption can be balanced to gain considerable cost saving in query execution. Thus, we conclude that the proposed framework is a necessary complement for the classic filter-ordering problem for actual applications. In future, we will further study the optimization of query planning with uncertainty in the query-filter structure, and the case that data items are highly correlated. 


\section{REFERENCES}

[1] B. Babcock, S. Babu, M. Datar, R. Motwani, and J. Widom, "Models and issues in data stream systems," in Proceedings of the 21st ACM SIGMOD-SIGACT-SIGART symposium on Principles of database systems, 2002, pp. 1-16.

[2] A. Koski, M. Juhola, and M. Meriste, "Syntactic recognition of ecg signals by attributed finite automata," Pattern Recognition, vol. 28, pp. 1927-1940, 1995.

[3] H. Wu, B. Salzberg, and D. Zhang, "Online event-driven subsequence matching over financial data streams," in Proceedings of the ACM SIGMOD, 2004, pp. 23-34.

[4] K. Munagala, U. Srivastava, and J. Widom, "Optimization of continuous queries with shared expensive filters," in Proceedings of the ACM SIGMOD-SIGACT-SIGART symposium on Principles of database systems, 2007, pp. 215-224.

[5] Z. Liu, S. Parthasarathy, A. Ranganathan, and H. Yang, "Nearoptimal algorithms for shared filter evaluation in data stream systems," in Proceedings of the ACM SIGMOD, 2008, pp. 133-146.

[6] V. V. Vazirani, Approximation algorithms. Springer, 2004.

[7] M. K. Aguilera, R. E. Strom, D. C. Sturman, M. Astley, and T. D. Chandra, "Matching events in a content-based subscription system," in Proceedings of the 18th annual ACM symposium on Principles of distributed computing, 1999, pp. 53-61.

[8] G. Cugola, E. Di Nitto, and A. Fuggetta, "Exploiting an event-based infrastructure to develop complex distributed systems," in Proceedings of the International Conference on Software Engineering, 1998.

[9] F. Fabret, H. A. Jacobsen, F. Llirbat, J. Pereira, K. A. Ross, and D. Shasha, "Filtering algorithms and implementation for very fast publish/subscribe systems," in ACM SIGMOD Record, 2001, pp. $115-126$.

[10] T. W. Yan and H. Garcia-Molina, "The sift information dissemination system," ACM Transactions on Database Systems, vol. 24, pp. 529$565,1999$.

[11] S. Finkelstein, "Common expression analysis in database applications," in Proceedings of the 1982 ACM SIGMOD, 1982.

[12] J. Park and A. Segev, "Using common subexpressions to optimize multiple queries," in Proceedings of the ICDE, 1988.

[13] S. Babu and J. Widom, "Continuous queries over data streams," $A C M$ SIGMOD Record, vol. 30, pp. 109-120, 2001.

[14] H.-S. Lim, J.-G. Lee, M.-J. Lee, K.-Y. Whang, and I.-Y. Song, "Continuous query processing in data streams using duality of data and queries," in Proceeding of ACM SIGMOD, 2006, pp. 313-324.

[15] S. Madden, M. Shah, J. M. Hellerstein, and V. Raman, "Continuously adaptive continuous queries over streams," in Proceedings of the ACM SIGMOD, 2002, pp. 49-60.

[16] L. Gao and X. S. Wang, "Continually evaluating similarity-based pattern queries on a streaming time series," in Proceedings of the ACM SIGMOD, 2002, pp. 370-381.

[17] H.-S. Lim, K.-Y. Whang, and Y.-S. Moon, "Similar sequence matching supporting variable-length and variable-tolerance continuous queries on time-series data stream," Information Sciences, vol. 178, pp. 1461-1478, 2007.

[18] P. Roy, S. Seshadri, S. Sudarshan, and S. Bhobe, "Efficient and extensible algorithms for multi query optimization," ACM SIGMOD Record, vol. 29, pp. 249-260, 2000.

[19] L. Golab and M. T. Özsu, "Processing sliding window multi-joins in continuous queries over data streams," in Proceedings of the VLDB, 2003, pp. 500-511.

[20] S. D. Viglas, J. F. Naughton, and J. Burger, "Maximizing the output rate of multi-way join queries over streaming information sources," in Proceedings of the VLDB, 2003, pp. 285-296.

[21] A. Arasu and J. Widom, "Resource sharing in continuous slidingwindow aggregates," in Proceedings of the VLDB, 2004, pp. 336347.

[22] Y. Zhu, E. A. Rundensteiner, and G. T. Heineman, "Dynamic plan migration for continuous queries over data streams," in Proceedings of the ACM SIGMOD, 2004, pp. 431-442.

[23] S. Babu, R. Motwani, K. Munagala, I. Nishizawa, and J. Widom, "Adaptive ordering of pipelined stream filters," in Proceedings of the ACM SIGMOD, 2004, pp. 407-418.

[24] A. Condon, A. Deshpande, L. Hellerstein, and N. Wu, "Flow algorithms for two pipelined filter ordering problems," in Proceedings of the ACM SIGMOD-SIGACT-SIGART symposium on Principles of database systems, 2006, pp. 193-202.
[25] K. Munagala, S. Babu, R. Motwani, and J. Widom, "The pipelined set cover problem," in Database Theory-ICDT. Springer, 2005, pp. 83-98.

[26] C. Olston, J. Jiang, and J. Widom, "Adaptive filters for continuous queries over distributed data streams," in Proceedings of the ACM SIGMOD , 2003, pp. 563-574.

[27] S. Rampone, "Probability-driven greedy algorithms for set cover," in VIII SIGEF Congress New Logics for the New Economy", 2001.

[28] M. Goemans and J. Vondrák, "Stochastic covering and adaptivity," in LATIN 2006: Theoretical informatics. Springer, 2006, pp. 532-543.

[29] J. a. Gama, I. Žliobaité, A. Bifet, M. Pechenizkiy, and A. Bouchachia, "A survey on concept drift adaptation," ACM Computing Surveys, vol. 46, no. 4, pp. 44:1-44:37, Mar. 2014.

[30] A. A. Qahtan, B. Alharbi, S. Wang, and X. Zhang, "A pca-based change detection framework for multidimensional data streams: Change detection in multidimensional data streams," in Proceedings of the ACM SIGKDD , 2015, pp. 935-944.

[31] E. B. Wilson, "Probable inference, the law of succession, and statistical inference," Journal of the American Statistical Association, vol. 22, pp. 209-212, 1927.

[32] S. Bischof, A. Karapantelakis, C.-S. Nechifor, A. Sheth, A. Mileo, and P. Barnaghi, "Semantic modeling of smart city data," in Proceedings of the W3C Workshop on the Web of Things: Enablers and services for an open Web of Devices, 2014.

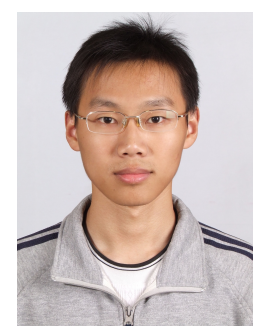

Qing Xie received the $B E$ degree in information science from University of Science and Technology of China in 2008, and the $\mathrm{PhD}$ degree in computer science from the University of Queensland, Australia, in 2013. $\mathrm{He}$ is currently a postdoctoral research fellow in Division of CEMSE, King Abdullah University of Science and Technology. His research interests include stream data mining, time series databases, continuous query optimization and e-Health.

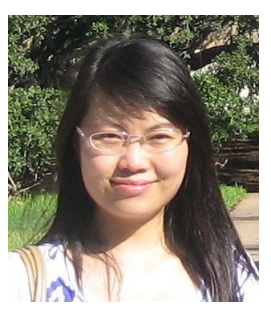

Xiangliang Zhang is currently Assistant Professor and directs the Machine Intelligence and kNowledge Engineering (MINE) Laboratory in King Abdullah University of Science and Technology (KAUST), Saudi Arabia. She earned her Ph.D. degree in computer science from INRIA-Université ParisSud, France in 2010. Her main research interests and experiences are in machine learning, data mining, and cloud computing.

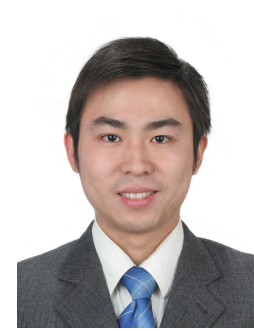

Zhixu Li is currently an associate professor at the Research Center on Advanced Data Analytics (ADA) in Soochow University, China. He used to work as a research fellow at King Abdullah University of Science and Technology (KAUST). He received his Ph.D. degree from the University of Queensland in 2013, and his B.S. and M.S. degree from Renmin University of China in 2006 and 2009 respectively. His research interests are Data Cleaning, Big Data Applications, Information Extraction and Information Retrieval.

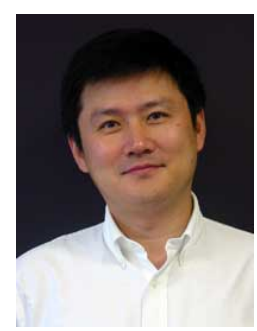

Xiaofang Zhou is a Professor of computer science with The University of Queensland. $\mathrm{He}$ is the Head of the Data and Knowledge Engineering Research Division. He is specially appointed Adjunct Professor under the Chinese National Qianren Scheme hosted by Renmin University of China (2010-2013), and by Soochow University since July 2013 where he leads the Research Center on Advanced Data Analytics (ADA). 